\title{
Correlated orientational and translational motions in supercooled liquids
}

\author{
Sarika Bhattacharyya, Arnab Mukherjee, and Biman Bagchi* \\ Solid State and Structural Chemistry Unit, Indian Institute of Science, Bangalore, India 560012
}

\begin{abstract}
We have carried out NPT molecular dynamics simulations of isolated ellipsoids in a glass forming binary mixture to gain insight into the nature of orientational relaxation (OR) in a viscous liquid. At high pressures when the liquid is highly viscous, the OR is found to occur mainly via correlated hopping, sometimes involving participation of several neighboring atoms, placed in a ring like tunnel. In the glassy state, hopping is found to be accompanied by larger fluctuations in the total energy and the volume of the system. Both orientational and translational hopping are found to be gated, restricted primarily by the entropic bottlenecks, with the orientational motion becoming increasingly slower than the translation as the pressure is increased. Orientational relaxation is found to occur with a wide distribution of decay times.
\end{abstract}

\section{INTRODUCTION}

Relaxation in supercooled liquids is now a subject of intense research. It is now recognized that anomalies in the relaxation in deeply supercooled liquids arise from an interplay between the dynamical cooperativity between different regions in the liquid (an idea originally introduced by Adam and Gibbs $^{1}$ ) and the influence of the underlying free energy surface of the liquid which increasingly exerts its influence as the glass transition region is approached ${ }^{2,3}$ from above. The essence of the dynamical cooperativity can arguably be described by the mode coupling theory ${ }^{4,5}$ which, however, fails to connect to the free energy landscape. There is still no comprehensive treatment of the cross-over from the dynamically cooperative region to the hopping dominated region. The cross-over scenario is intimately connected with other concepts, like the fragility and the super-Arrhenius behavior of the supercooled liquid.

Orientational relaxation in glassy liquid has proved to be the most powerful probe of the dynamics. The dielectric relaxation study by Goldstein and Johari first revealed the emergence of the $\alpha-\beta$ bifurcation and the disappearance of the $\alpha$ relaxation, as the glass transition is approached from higher temperature. ${ }^{6}$ The NMR studies of Ediger and coworkers find an emergence of a new time scale near the glass transition temperature. ${ }^{7}$ This new time scale is larger than $\alpha$ relaxation time. Ediger and other workers also found evidence of heterogeneity and decoupling of translational and rotational motions. ${ }^{7,8}$

Theoretical and computer simulation studies have mostly been directed towards understanding spherical systems, like the Lennard-Jones and the hard spheres. In this study, we report detailed MD [constant pressure $(P)$, temperature $(T)$, and constant total number of particles $(N)]$ simulations of orientational relaxation of a few isolated ellipsoids in glass forming binary liquids. The questions we address here are the following: (a) How are the orientational and the transla-

*Electronic mail: bbagchi@sscu.iisc.ernet.in tional motions correlated? (b) What is the energy surface for orientational hopping? (c) How local is the hopping phenomenon? Or in other words, how relevant is the free energy landscape?

The organization of the rest of the article is as follows: In the next section we present the systems and the simulation details. The results and discussions are presented in Sec. III. In the concluding section (Sec. IV) we summarize the results.

\section{SYSTEM AND SIMULATION DETAILS}

Our solvent is represented by Kob-Andersen (KA) binary mixture ${ }^{9}$ which is known to be a good glass former and has been extensively studied, ${ }^{10}$ and our solute probes are prolate ellipsoids. Pressure is kept constant by Andersen's piston method ${ }^{11}$ while in the case of temperature, a damped oscillator method has been adopted which keeps temperature constant at each and every time step. ${ }^{12}$ The piston mass involved here is $0.0027\left(m_{A} / \sigma_{A}^{4}\right)$ which is regarded as optimum. $^{12,13}$ The interactions between the particles are modeled by different potentials. The interaction between the spheres are given by Lennard-Jones Potential (as in the KA Model) and the interaction between two ellipsoids with arbitrary orientations is assumed to be given by the Gay-Berne (GB) potential, ${ }^{14}$

$$
\begin{aligned}
U_{\mathrm{GB}}= & 4 \epsilon\left(\hat{r}, \hat{u}_{1}, \hat{u}_{2}\right)\left[\left(\frac{\sigma_{0}}{r-\sigma\left(\hat{r}, \hat{u}_{1}, \hat{u}_{2}\right)+\sigma_{0}}\right)^{12}\right. \\
& \left.-\left(\frac{\sigma_{0}}{r-\sigma\left(\hat{r}, \hat{u}_{1}, \hat{u}_{2}\right)+\sigma_{0}}\right)^{6}\right],
\end{aligned}
$$

where $\hat{u}_{1}, \hat{u}_{2}$ are the axial vectors of the ellipsoids 1 and 2. $\hat{r}$ is the vector along the intermolecular vector $r=r_{2}-r_{1}$, where $r_{1}$ and $r_{2}$ denote the centers of mass of ellipsoids 1 and 2 , respectively. $\sigma\left(\hat{r}, \hat{u}_{1}, \hat{u}_{2}\right)$ and $\epsilon\left(\hat{r}, \hat{u}_{1}, \hat{u}_{2}\right)$ are the orientation-dependent range and strength parameters, respectively, and $\sigma$ and $\epsilon$ depend on the aspect ratio $\kappa$. Finally, the 
interaction between a sphere and an ellipsoid is accounted for by a modified GB-LJ potential given below: ${ }^{16}$

$$
U_{E i}=4 \epsilon_{E i}\left[\left(\frac{\sigma(\theta)_{E i}}{r}\right)^{12}-\left(\frac{\sigma(\theta)_{E i}}{r}\right)^{6}\right],
$$

where " $E$ " denotes the ellipsoids and " $i$ " can be " $A$ " or " $B$." The expression for $\sigma(\theta)_{E i}$ is available. ${ }^{15,16}$

The ellipsoid in a binary mixture system with the above mentioned potential is a well behaved system and it can also exhibit the experimentally observed anomalous viscosity dependence of the orientational correlation time ${ }^{15}$ Four ellipsoids were placed far from each other in a binary mixture of 500 particles with number of " $A$ " particles, $N_{A}=400$ and number of " $B$ " type particles $N_{B}=100$. The reduced temperature is expressed as $T^{*}\left(=k_{B} T / \epsilon_{A A}\right)$, where $k_{B}$ is the Boltzmann constant, the reduced pressure as $P^{*}$ $\left(=P \sigma_{A}^{3} / \epsilon_{A A}\right)$ and the reduced density as $\rho^{*}\left(=\rho \sigma_{A}^{3}\right)$. The time is scaled by $\sqrt{\left(m_{A} \sigma_{A}^{2} / \epsilon_{A A}\right)}$. The time step of the simulation is $0.002 \tau$ and the system is equilibrated for $1.5 \times 10^{5}$ steps and the data collection steps varied from $1 \times 10^{6}$ to $5 \times 10^{6}$.

\section{RESULTS AND DISCUSSIONS}

Systematic simulations of the model system (at $T^{*}$ $=0.8$ ) have been carried out by varying pressure over a large range to study the system from its normal liquid regime to the glassy state. The dynamical nature of any system can be characterized from the study of the relaxation of the dynamical correlations, the value of the transport properties and also from an analysis of the trajectories of the particles. In the present study we characterized the system from the value of the viscosity, and the nature of the translational motion of all the particles and orientational motion of the ellipsoids. The viscosity has been calculated by integrating the stress autotime correlation function and the nature of the translational and orientational trajectories are analyzed from the normalized probability distribution for spatial displacement, $P^{i}(r, t)=\left(1 / N_{i}\right) r^{2} \Sigma_{j=1}^{N_{i}} \delta\left(r-r_{j}(t)\right)$, and for angular displacement, $P(\theta, t)=1 / N_{E} \sum_{j=1}^{N_{E}} \delta\left(\theta-\theta_{j}(t)\right)$. Here $N_{i}$ is the number of particles of the " $i$ " kind where " $i$ " can be $A, B$ or E.

At $P^{*}=2.0$, the system is in the normal liquid regime. Here the reduced density is 1.13 and the reduced viscosity is 13.446. The motion is continuous and orientational correlation decays like in a normal liquid. Both the normalized probability distributions for spatial and angular displacement are Gaussian in the long time. This behavior changes as pressure is increased.

At $P^{*}=5$ where $\eta^{*}=479.5$, it is a viscous liquid. In Fig. 1 we plot $P(\theta, t)$ at pressures 5 and 6 . At $P^{*}=5$, the rotational motion is mostly continuous and at long time $P(\theta, t)$ is Gaussian. However, at $P^{*}=6$ viscosity $\eta$ is about 2340. At long time $P(\theta, t)$ has one peak at small angle and there is also another peak between $160^{\circ}-180^{\circ}$. Typical orientational trajectories at pressure 5 and 6 are shown in Figs. 2(a) and 2(b), respectively. At pressure 5 the orientation changes continuously whereas at pressure 6 the ellipsoids undergo sharp $180^{\circ}$ rotation as shown in Fig. 2(b). We have

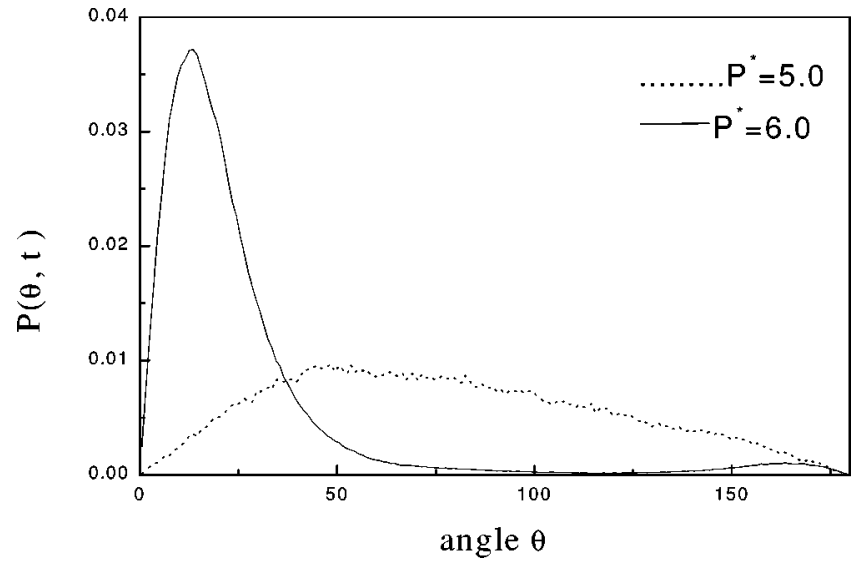

FIG. 1. Dashed and solid lines show variation of $P(\theta, t)$ for $t=200 \tau$, against angular displacement $\theta$ for pressure $P^{*}=5$ and $P^{*}=6$, respectively. At $P^{*}=6$ the distribution is bimodal with a second peak appearing around $160^{\circ}$.

analyzed the trajectories of spatial displacement of the ellipsoid and its neighbors during the period of orientational hopping. We found that the ellipsoid almost retains its position while performing this orientational hopping and about 7-8 of its neighbors have a shift in their average positions by about $0.2-0.8 \sigma$ and then once the orientational hopping is over they come back to their old positions.

Note that the second peak of $P(\theta, t)$ is shifted a little from $180^{\circ}$. The plot (not shown here) of the self-part of the angular van Hove function $G_{s}(\theta, t) \propto P(\theta, t) / \sin \theta$, shows a peak at $180^{\circ}$, but $G_{s}(\theta, t)$ also predicts this second peak even at pressure 5 where there is no signature of $180^{\circ}$ hopping [as shown in Fig. 2(a)]. The second peak in $G_{s}(\theta, t)$, therefore, arises partly from the $1 / \sin (\theta)$ factor and is not a conclusive evidence of orientational hopping by $180^{\circ}$. Also note that at higher pressure the orientational hopping is not always $180^{\circ}$ rotation but can be a sudden rotation by a smaller angle as presented later in the discussion on the simulation results at $P^{*}=10$.

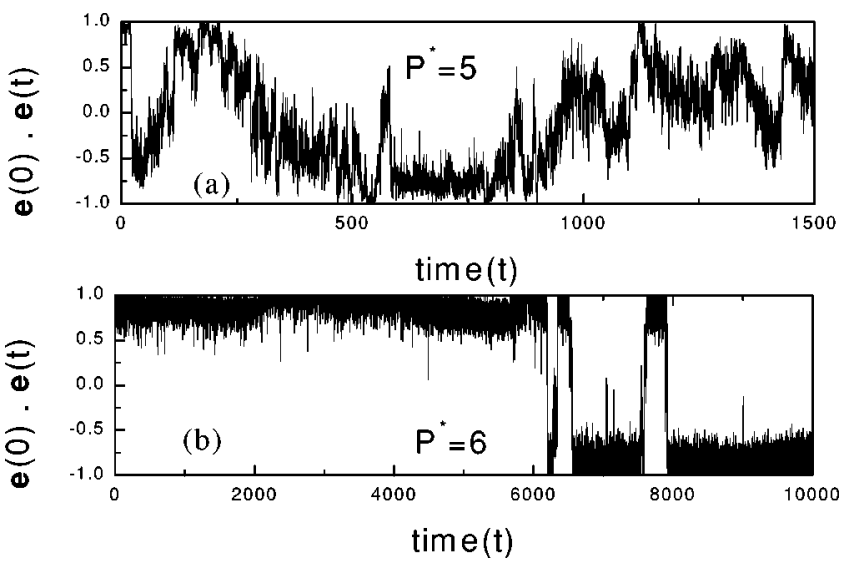

FIG. 2. (a) Dot product of the instantaneous orientation and the orientation at $t=0$ of an ellipsoid, plotted against reduced time at $P^{*}=5.0$ The orientation of the ellipsoid changes continuously. (b) The same as (a) but at $P^{*}$ $=6$. The orientation of the ellipsoid at this pressure changes mostly by $180^{\circ}$ hops. 

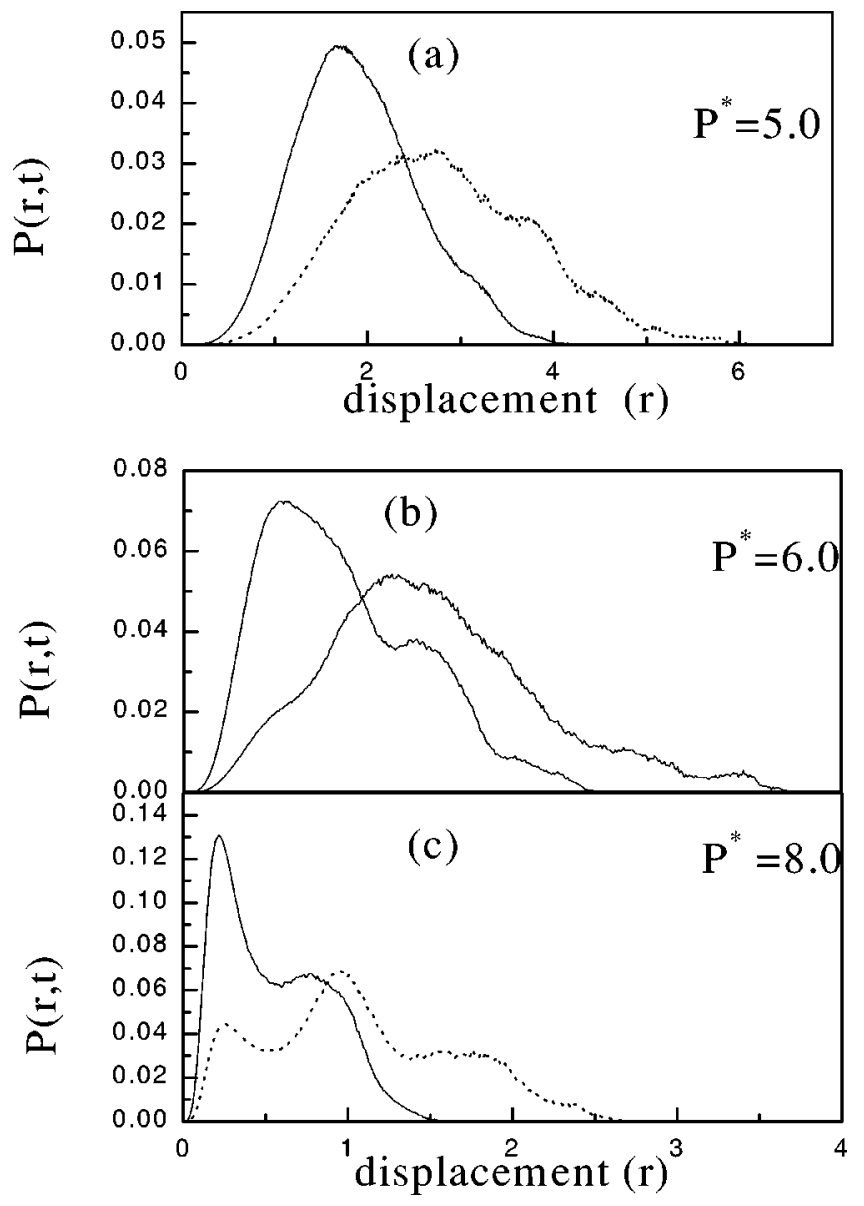

FIG. 3. (a) Distribution of $P(r, t)$ against scaled displacement $(r)$ at $t$ $=500$ for two different types of particles (" $A$ " and " $B$ ") at $P^{*}=5.0$. A solid line and a dashed line denote bigger and smaller particles, respectively. (b) The same plot as (a), at $P^{*}=6.0$. (c) The same plot as (a), at $P^{*}$ $=8.0$.

Figures 3(a), 3(b), and 3(c) show $P^{i}(r, t)$ at pressures, 5, 6 , and 8 , respectively. The solid and the dotted lines are for the larger $(A)$ and the smaller $(B)$ particles, respectively. While at $P^{*}=5$ both $P^{A}(r, t)$ and $P^{B}(r, t)$ are Gaussian, at pressure $6, P^{B}(r, t)$ is still Gaussian but $P^{A}(r, t)$ shows two peaks. This is because at this pressure although most of the small " $B$ " particles still have continuous motion with some amount of hopping, the " $A$ " particles move primarily through hopping. Note that for translational motion we define a hop when a particle has a sudden displacement of at least $0.5 \sigma$ within $50 \tau$. At pressure 8 , both $P^{A}(r, t)$ and $P^{B}(r, t)$ show multiple peaks. At this pressure the density is around 1.36 and hopping is the dominant mode of transport for all the particles. A small peak observed in $P^{B}(r, t)$ at higher $r$ value $(2.5 \sigma)$ signifies correlated hopping. The peak at $0.2 \sigma$ describes the vibrational motion within the cage. We found that the cross-over from continuous to hopping dominated regime is accompanied by a dramatic slowdown in the decay of the stress auto-time correlation function and the sharp rise in the value of the viscosity. From the above analyses, we conclude that the larger $(A)$ particles freeze near $P^{*}=6$ while the smaller $(B)$ particles freeze near $P^{*}$ $=7$. Beyond $P^{*}=8$ we have not been able to calculate the value of viscosity since within the simulation timescale the

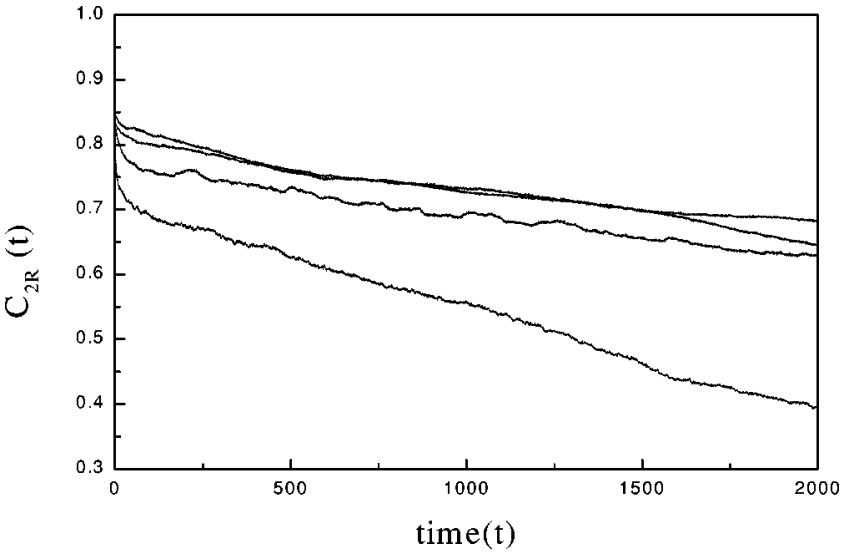

FIG. 4. Decay of $C_{2 R}(t)$ of four ellipsoids plotted against reduced time at $P^{*}=6$. The orientational relaxation time of the ellipsoids are heterogeneous.

long time tail of the stress autocorrelation function did not decay. The study of the OCF shows that at high pressures $\left(P^{*} \geqslant 6\right)$ the OCF decays only through hopping and there is a distribution of relaxation times. While the average orientational correlation function is nonexponential, the decay of each ellipsoid is found to be only weakly nonexponential, with widely different relaxation times as shown in Fig. 4 for $P^{*}=6$. Thus, the decay of orientational correlation function can truly be regarded to be heterogeneous at high pressure.

At even higher pressure $P^{*}=10$, the system is so densely packed that there hardly remains any signature of liquid like region. The reduced density of this system is 1.41 where each ellipsoid has about 22 neighbors. In this highly dense system although the stress correlation does not decay the study of the orientational and translational motion of the ellipsoids becomes important and remains the only tool to probe the liquid dynamics. At this pressure, hopping of the ellipsoid is indeed a rare event. Those which are moving are found to have primarily two different kinds of motion: One is a many particle correlated hopping and the other motion is a motion in a ring like tunnel as has been observed earlier by other authors in different systems. ${ }^{17}$

Correlated orientational and translational hoppings have been observed. Figures 5 and 6 show the displacement of the 1 st and 2nd ellipsoid in reduced time and the change in their instantaneous orientation. A study of the first ellipsoid and its neighboring particle dynamics reveal that only about 5-6 particles translate (greater than $0.525 \sigma$ ) during (or just prior to) the time the ellipsoid hops. This has been shown in Fig. 7. The OCF decays a considerable amount only during this period. Analyzing the dynamics of the second ellipsoid and its neighboring particles and their neighbors, we found that 5 particles (including the 2 nd ellipsoid) have similar trajectories (significant displacement, all around 1650 $\tau$ ). All these particles are not the neighbors of the ellipsoid. Instead, they are the consecutive neighbors and are placed in a ring like tunnel. Thus although the ellipsoid translates a considerable amount in this tunnel, the orientational dynamics is constrained, leading to less decay of the OCF (only up to 0.82).

We also find that hopping of the particle is associated with a larger fluctuation in volume and total energy in this glassy system. Note that although the correlation length of 

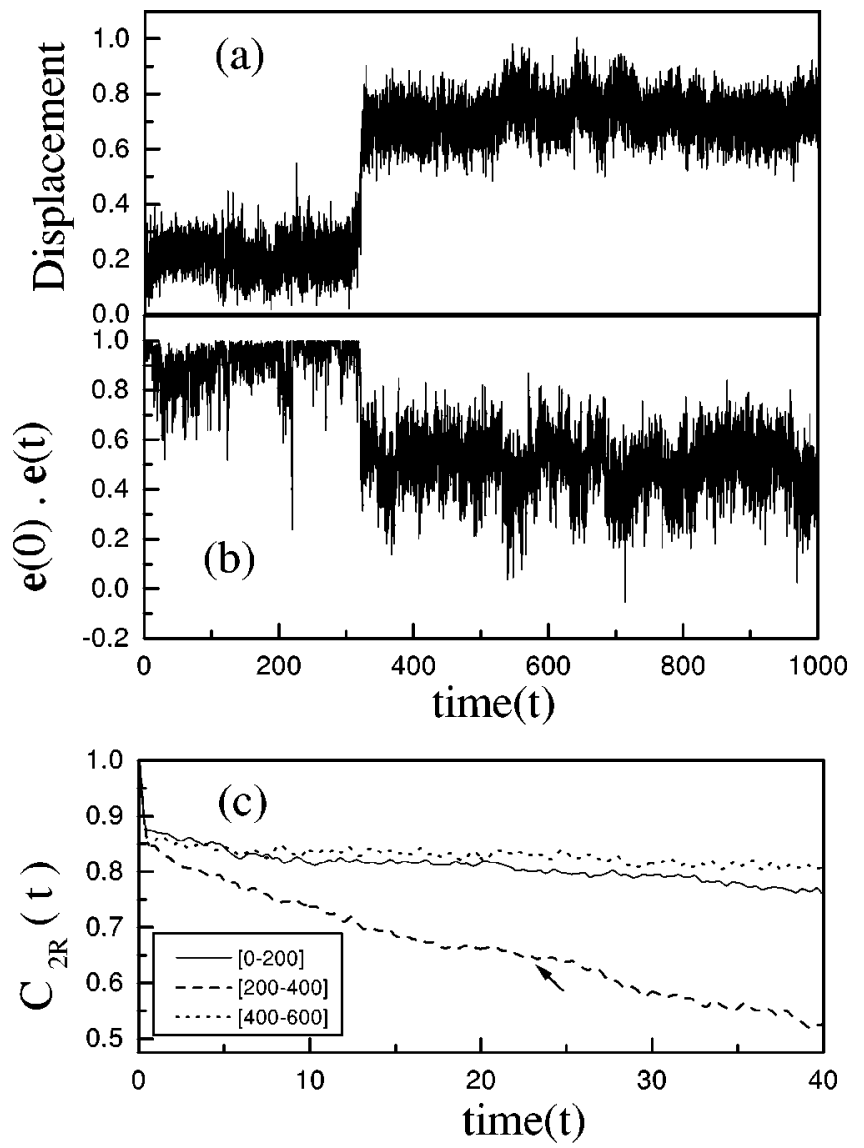

FIG. 5. (a) Displacement of an ellipsoid (tag 1) occurs only by hopping at around $300 \tau$. (b) Dot product of instantaneous orientation and the orientation at the time $t=0$ shows that orientational and translational hopping happen at the same time. (c) The decay of $C_{2 R}(t)$ in different time intervals is plotted. The arrow points to the OCF which shows prominent decay during the time interval of the hopping. The plots are at $P^{*}=10$.

the system increases at this high pressure, the ellipsoids can still be treated as isolated from one another. This can be justified from the study of the trajectories of the ellipsoids in the same run. While one of them jumps, the motion of the others remain unperturbed and they only perform vibrational motion about their mean positions.

In an ordinary liquid rotational relaxation time, $\tau_{R}$, is of the order of $10^{-11} \mathrm{~s}$ whereas the time taken to translate 1 molecular diameter, $\tau_{T}$, is about $10^{-10} \mathrm{~s}$. In a supercooled liquid both translation and rotation slows down. At $P^{*}$ $=10$, since the orientational correlation function does not decay much, it is rather difficult to make an estimate of $\tau_{R}$. We found that even at $1200 \tau$ the OCF does not decay below 0.55 . Thus it can be safely concluded that in our system $\tau_{2 R} \gg \tau_{T}$. This observation is similar to that reported by Ediger and co-workers where they found that the molecule translates hundreds of molecular diameter before it rotates once. $^{7}$

Figure 8 shows the variation of the single particle potential energy during hopping as a function of time, both for the ellipsoid and two of its neighbors. We have also calculated the total energy of the ellipsoid and its neighbors during the period of hopping. We found that there is also no change in this total energy. From our simulation results we have esti-
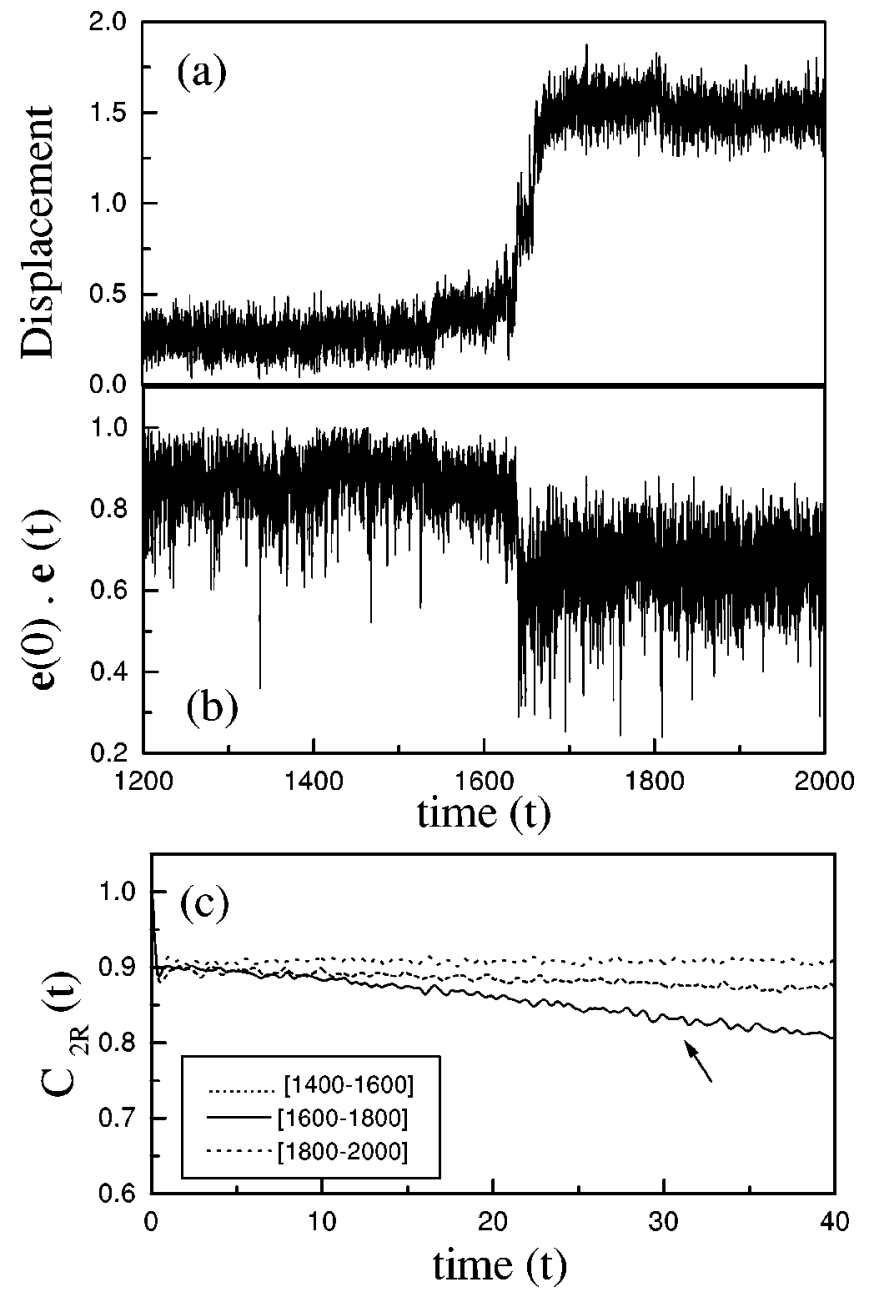

FIG. 6. (a) Displacement of another ellipsoid (tag 2) where it undergoes motion in a ring like tunnel. (b) Dot product of the instantaneous orientation and the orientation at time $t=0$. Both the orientational hopping and translational motion are correlated. (c) The decay of $C_{2 R}(t)$ in different time intervals are plotted. Although, the ellipsoid translates about $1 \sigma$ distance, due to constrained orientational dynamics in the ring like tunnel the OCF decays only up to 0.82 . The arrow points to the OCF, obtained during the period of hopping. The plots are at $P^{*}=10$.

mated the hopping rate to be $2 \times 10^{7}$. According to the transition state theory the rate of hopping can be written as ${ }^{18}$

$$
k=\frac{k_{B} T}{h} e^{\Delta S / k_{B}} e^{-\Delta E / k_{B} T},
$$

where $k$ is the rate of hopping, $h$ is the Planck constant, $\Delta S$ and $\Delta E$ are the average change of entropy and energy per hopping, respectively. In Eq. (3) if we put the calculated hopping rate and also consider that there is no energy cost due to hopping, then the estimated change in entropy per hopping is about $11-12 k_{B} T$. Thus a study of the energetics (during orientational and translational hopping) reveals that both kinds of hopping are a gated process, where the free energy barrier (or the saddle) is entropic in nature. The ellipsoid hopping is associated with instantaneous rearrangement (significant displacement) of surrounding molecules. At high pressure the particles are densely packed and trapped in regions separated by small windows (entropic bottlenecks). ${ }^{19}$ If the motion of the ellipsoid is in the $x$-direction then the sur- 


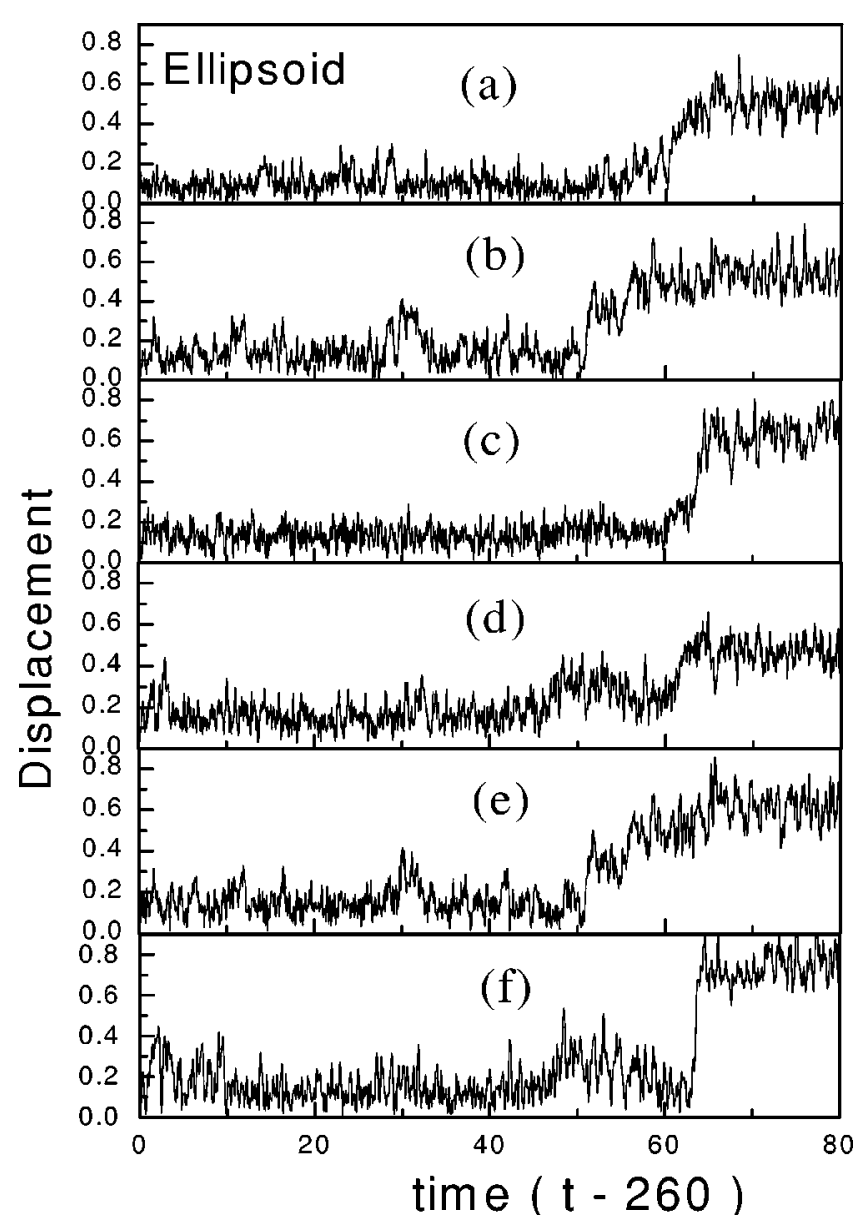

FIG. 7. The spatial displacement of the ellipsoid (tag 1) and 5 of its neighbors between $260 \tau$ and $340 \tau$, at $P^{*}=10$. (a) The displacement of the ellipsoid. (b),(c),(d),(e) Displacements of those neighbors which are larger in size (" $A$ " particles) and (f) is that for a smaller particle (" $B$ " particle). There is collective hopping of the ellipsoid and a few of its neighbors.

rounding particles are found to move in the perpendicular plane. Thus only rearrangements or displacement of surrounding molecules open up the gate and allow the ellipsoid to move as envisaged in the Zwanzig model. ${ }^{19}$ In the process of creation of the gate some of the particles register a small rise in energy [shown in Figs. 4(b) and 4(c)]. However, as mentioned above, the sum of energies of the ellipsoid and its neighbors remains the same.

\section{CONCLUSION}

In this article we have presented a study of the translational and rotational dynamics of isolated ellipsoids in a glass forming binary mixture. The study has been performed at various pressures, at a constant temperature. The system at different state points have been characterized from the value of the viscosity and also from the probability distribution of the spatial and angular displacements. As expected, we find that the larger particles start hopping at a lower pressure, where the smaller particles still move continuously. Beyond pressure 6 the orientational relaxation shows a heterogeneous relaxation time scale.

At very high pressure, $P^{*}=10$, all the particles move only via hopping, where hopping itself becomes a rare event.

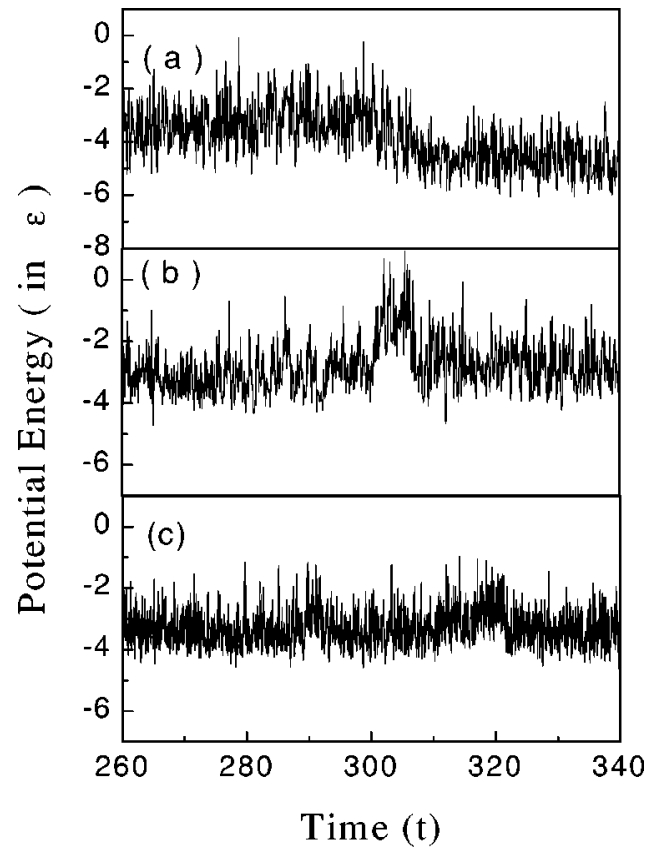

FIG. 8. (a) Variation of the single particle potential energy (SPPE) of the ellipsoid with time around hopping signifies gated diffusion. (b) and (c) show the variation of SPPE of two neighboring particles of the above ellipsoid. The plots are at $P^{*}=10$.

At this pressure the stress auto-correlation function, the orientational correlation function and all other dynamical correlations cease to decay within the simulation timescale. Thus, the study of the microscopic motion remains the only tool and indeed the translational and orientational trajectories of the ellipsoids reveal some interesting features. We have primarily observed two different kinds of motion. One is the motion of the ellipsoid in a ring like tunnel and the other is the correlated hopping of the ellipsoid along with 5-6 of its neighboring particles. The study also shows that orientational and translational motions are correlated in time. The orientational relaxation of the ellipsoid can only take place when there is a collective motion of several particles which are either placed in a ring or are the nearest neighbors of the ellipsoid. There is clearly a waiting time distribution of this collective event to happen and it is expected to play an important role in the relaxation of glassy liquids. A complete theory of these highly collective processes would be nontrivial, and would perhaps first require a "coarse-grained" theory of the type discussed by Xia and Wolynes. ${ }^{20}$

The study of the energetics during the period of translational and orientational hopping predicts that both are gated process where the free energy barrier is entropic in nature.

We also find that as the glass transition is approached, the orientational relaxation occurs increasingly on a slower time scale, compared to translation motion. This is because molecules can hop preserving its orientation and also $180^{\circ}$ orientational hopping does not change 2nd rank harmonics, often probed in experiments. In the glassy state the hopping of ellipsoid is local. Finally, we note that the present simulations can reproduce several behaviors observed in recent experiments on orientational relaxation in deeply supercooled liquids. 


\section{ACKNOWLEDGMENTS}

It is a pleasure to thank Professor C. Austen Angell, Professor Peter Wolynes, and Professor Srikanth Sastry for discussions and correspondence. The work has been supported in part by grants from the Council of Scientific and Industrial Research and the Department of Science and Technology, India.

${ }^{1}$ G. Adam and J. H. Gibbs, J. Chem. Phys. 43, 139 (1965).

${ }^{2}$ F. H. Stillinger and T. A. Weber, Phys. Rev. A 25, 978 (1982); Science 225, 983 (1984).

${ }^{3}$ R. Zwanzig, J. Chem. Phys. 79, 4507 (1983); E. Rabani, J. D. Gezelter, and B. J. Berne, Phys. Rev. Lett. 82, 3649 (1999); T. Keyes, G. V. Vijayadamodar, and U. Zurcher, J. Chem. Phys. 106, 4651 (1997).

${ }^{4}$ W. Gotze, in Liquids, Freezing and the Glass Transition, edited by D. Levesque, J. P. Hansen, and J. Zinn-Justin (North-Holland, Amsterdam, 1990); W. Gotze and L. Sjogren, Phys. Rev. A 43, 5442 (1991).

${ }^{5}$ B. Bagchi and S. Bhattacharyya, Adv. Chem. Phys. 116, 67 (2001).

${ }^{6}$ G. P. Johari and M. Goldstein, J. Chem. Phys. 53, 2372 (1970); 55, 4245 (1971).
${ }^{7}$ M. D. Ediger, C. A. Angell, and S. R. Nagel, J. Phys. Chem. 100, 13200 (1996); M. T. Cicerone and M. D. Ediger, J. Chem. Phys. 104, 7210 (1996); 103, 5684 (1995).

${ }^{8}$ G. Heuberger and H. Sillescu, J. Phys. Chem. 100, 15255 (1996).

${ }^{9}$ W. Kob and H. C. Andersen, Phys. Rev. E 51, 4626 (1995); W. Kob and H. C. Andersen, Phys. Rev. Lett. 73, 1376 (1994).

${ }^{10}$ S. Sastry, P. G. Debenedetti, and F. H. Stillinger, Nature (London) 393, 554 (1998); S. Sastry, Phys. Rev. Lett. 85, 590 (2000); S. Sastry, Nature (London) 409, 164 (2001).

${ }^{11}$ H. C. Andersen et al., "Rapport d'activite scientifique du CECAM," Secs. 7.4, 7.4.3, pp. 82-115.

${ }^{12}$ D. Brown and J. H. R. Clarke, Mol. Phys. 51, 1243 (1984).

${ }^{13}$ J. M. Haile and H. W. Graben, J. Chem. Phys. 73, 2412 (1980).

${ }^{14}$ J. G. Gay and B. J. Berne, J. Chem. Phys. 74, 3316 (1981).

${ }^{15}$ S. Bhattacharyya and B. Bagchi, J. Chem. Phys. 115, 9065 (2001).

${ }^{16}$ S. Ravichandran and B. Bagchi, J. Chem. Phys. 111, 7505 (1999).

${ }^{17}$ C. Donati et al., Phys. Rev. Lett. 80, 2338 (1998).

${ }^{18}$ B. Bagchi, Int. Rev. Phys. Chem. 6, 1 (1987).

${ }^{19}$ H.-X. Zhou and R. Zwanzig, J. Chem. Phys. 94, 6147 (1991); J. Machta and R. Zwanzig, Phys. Rev. Lett. 50, 1959 (1983).

${ }^{20}$ X. Xia and P. G. Wolynes, Phys. Rev. Lett. 86, 5526 (2001). 